\title{
Diacronie
}

Studi di Storia Contemporanea

$\mathrm{N}^{\circ} 36,4 \mid 2018$

Viaggi e turismo nell'Europa del Novecento

\section{Turismo, disabilità, inclusione: le colonie estive dell'Istituto dei Ciechi di Milano nel secondo Novecento}

\section{Luciano Maffi e Martino Lorenzo Fagnani}

\author{
(2) OpenEdition \\ Journals \\ Edizione digitale \\ URL: https://journals.openedition.org/diacronie/10123 \\ DOI: 10.4000/diacronie. 10123 \\ ISSN: 2038-0925 \\ Editore \\ Association culturelle Diacronie \\ Notizia bibliografica digitale \\ Luciano Maffi e Martino Lorenzo Fagnani, «Turismo, disabilità, inclusione: le colonie estive dell'Istituto \\ dei Ciechi di Milano nel secondo Novecento», Diacronie [Online], № 36, 4 | 2018, documento 5, online \\ dal 29 décembre 2018, consultato il 17 février 2022. URL: http://journals.openedition.org/diacronie/ \\ 10123 ; DOI: https://doi.org/10.4000/diacronie.10123
}




\title{
Diacronie
}

Studi di Storia Contemporanea

$36,4 / 2018$

Viaggi e turismo nell'Europa del Novecento

\section{Turismo, disabilità, inclusione: le colonie estive dell'Istituto dei Ciechi di Milano nel secondo Novecento}

\author{
Luciano MAFFI
}

Martino Lorenzo FAGNANI

Per citare questo articolo:

MAFFI, Luciano, FAGNANI, Martino Lorenzo, «Turismo, disabilità, inclusione: le colonie estive dell'Istituto dei Ciechi di Milano nel secondo Novecento», Diacronie. Studi di Storia Contemporanea : Viaggi e turismo nell'Europa del Novecento, $36,4 / 2018,29 / 12 / 2018$,

URL: < http://www.studistorici.com/2018/12/29/maffi-fagnani_numero_36/ >

Diacronie Studi di Storia Contemporanea $\rightarrow$ http://www.diacronie.it

Rivista storica online. Uscita trimestrale.

redazione.diacronie@hotmail.it

Comitato di direzione: Naor Ben-Yehoyada - João Fábio Bertonha - Christopher Denis-Delacour - Maximiliano Fuentes Codera Anders Granås Kjøstvedt - John Paul Newman - Deborah Paci - Niccolò Pianciola - Spyridon Ploumidis - Wilko Graf Von Hardenberg

Comitato di redazione: Jacopo Bassi - Luca Bufarale - Gianluca Canè - Luca G. Manenti - Fausto Pietrancosta - Alessandro Salvador - Matteo Tomasoni - Luca Zuccolo

Diritti: gli articoli di Diacronie. Studi di Storia Contemporanea sono pubblicati sotto licenza Creative Commons 3.0. Possono essere riprodotti e modificati a patto di indicare eventuali modifiche dei contenuti, di riconoscere la paternità dell'opera e di condividerla allo stesso modo. La citazione di estratti è comunque sempre autorizzata, nei limiti previsti dalla legge. 


\title{
5/ Turismo, disabilità, inclusione: le colonie estive dell'Istituto dei Ciechi di Milano nel secondo Novecento ${ }^{1}$
}

\author{
Luciano MAFFI
}

Martino Lorenzo FAGNANI

L'articolo si propone di analizzare il significativo spazio che la disabilità e la sua inclusione hanno nella storia del turismo nell'Italia novecentesca. Partendo da uno spoglio della normativa italiana riguardante i disabili e la loro inclusione nella società italiana contemporanea, si passa in un secondo tempo a considerare le tipologie di turismo che nel secolo scorso hanno consentito alle persone con disabilità di viaggiare in un contesto di sicurezza e di relativa tranquillità. Cuore dell'articolo è il case study proposto: le colonie estive per i bambini e i ragazzi ospiti dell'Istituto dei Ciechi di Milano, con particolare riguardo al periodo di grandi cambiamenti da metà anni Cinquanta a metà anni Settanta. Tramite documenti inediti, si analizzano l'organizzazione e l'aspetto ricreativo dei giovani in soggiorni di mare e di montagna.

\section{Un quadro introduttivo}

La WHO (World Health Organization) precisa nel World Report on Disability del 2011 che la disabilità fa parte della condizione umana e che quasi tutte le persone a un certo punto della loro vita si ritrovano a essere permanentemente o temporaneamente «impaired», se non altro avvicinandosi alla vecchiaia. Con riferimento all'International Classification of Functioning, Disability and Health, il Report fa riferimento alla disabilità come a un «umbrella term for impairments, activity limitations, and participation restrictions». Inoltre, citando la Convenzione sui diritti delle persone con disabilità adottata dalle Nazioni Unite nel 2006, sprona i governi e la società civile a promuovere, proteggere e garantire «the full and equal enjoyment of all human rights and fundamental freedoms by all persons with disabilities, and to promote respect for their inherent

\footnotetext{
${ }^{1}$ Il presente articolo è frutto di una riflessione e di una ricerca archivista e storiografica comuni. Tuttavia, ai fini della valutazione i paragrafi 2, 3 e 6 sono da attribuire a Luciano Maffi, i paragrafi 1, 4 e 5 a Martino Lorenzo Fagnani, mentre gli autori hanno steso insieme il paragrafo 7.
} 
dignity», auspicando un impegno nazionale e internazionale. Il concetto di disabilità è ricondotto quindi agli aspetti negativi presenti nell'interazione tra persone che hanno una particolare condizione di salute e fattori di natura personale e ambientale (tra cui mezzi di trasporto ed edifici pubblici inaccessibili) ${ }^{2}$.

Quanto dichiarato dalla WHO è da intendersi come l'ufficializzazione di un rinnovato interesse a livello internazionale del rapporto tra disabilità e sfera sociale: interesse che ha conosciuto un importante sviluppo soprattutto nell'ultimo ventennio, da attribuire principalmente alla mutata sensibilità nei confronti di questa tematica e alla crescente consapevolezza che l'inclusione è un principio fondamentale del vivere civile. Quest'ultimo approccio è stato motivato dal varo progressivo di leggi adeguate in molti Stati, ma al contempo ha sollecitato esso stesso una normativa che potesse occuparsi nel modo più appropriato delle necessità delle persone disabili. Un bisogno reso ancora più urgente dal fatto che, in base a una stima della WHO di inizio 2018, circa il $15 \%$ della popolazione mondiale (quindi più di un miliardo di persone) ha una qualche forma di disabilità, dal che si conclude che ogni comunità abbia al proprio interno individui disabili ${ }^{3}$.

È tuttavia da precisare che, a fianco di un crescente numero di opere relative alla percezione della disabilità da una prospettiva storiografica ${ }^{4}$, attualmente la letteratura internazionale e italiana relativa all'inclusione e alla disabilità si è occupata soprattutto di ambiti come l'istruzione, il lavoro, l'accesso ai servizi e alle infrastrutture ${ }^{5}$. In misura assai minore è stato affrontato il legame tra i tipi di disabilità e le attività ricreative, compreso il fenomeno turistico,

2 WORLD HEALTH ORGANIZATION, World Report on Disability, Geneva, WHO Press, 2011, pp. 3-9. Si veda anche la seguente pagina del sito web della WHO: «Health topics: Disabilities», in World Health Organization, URL:

< http://www.who.int/topics/disabilities/en/ > [consultato il 2 ottobre 2018].

3 WORLD HEALTH ORGANIZATION, International Classification of Impairments, Disabilities and Handicaps (ICIDH), Genève, World Health Organization, 1997; «Disability and health», in World Health Organization, URL:

< http://www.who.int/en/news-room/fact-sheets/detail/disability-and-health > [consultato il 2 ottobre 2018]. A tal proposito è di grande interesse un dato presente in SMALL Jennie, DARCY, Simon, Tourism, disability and mobility, in COLE, Stroma, MORGAN, Nigel (eds.), Tourism and inequality. Problems and prospects, Wallingford (UK), CABI Publishing, 2010, pp. 1-20. A p. 1, infatti, è riportata la percentuale stimata dalle Nazioni Unite a inizio 2009, ovvero il 10\%. Tale crescita tiene conto anche dei legami con l'invecchiamento della popolazione mondiale e l'aumento delle malattie croniche, circostanze di cui la World Health Organization è consapevole.

4 Si citano alcune delle opere più autorevoli e aggiornate: REMBIS, Michael, KUDLICK, Catherine J., NIELSEN, Kim E. (eds.), The Oxford handbook of disability history, Oxford, Oxford University Press, 2018; HANES, Roy, BROWN, Ivan, HANSEN, Nancy E. (eds.), The Routledge history of disability, Abingdon, Routledge, 2017; NIELSEN, Kim E., A disability history of the United States, Boston, Beacon Press, 2012; SCHIANCHI, Matteo, Storia della disabilità. Dal castigo degli dèi alla crisi del welfare, Roma, Carocci, 2012.

5 In tali ambiti è importante citare: VILLE, Isabelle, FILLION, Emmanuelle, RAVAUD, Jean-François, Introduction à la sociologie du handicap. Histoire, politiques et expérience, Louvaine-la-Neuve, De Boeck, 2014; MEDEGHINI, Roberto, VALTELLINA, Enrico, Quale disabilità? Culture, modelli e processi di inclusione, Milano, FrancoAngeli, 2006; STIKER, Henri-Jacques, A history of disability, Ann Arbor, University of Michigan Press, 1999; SAFFORD, Philip L., SAFFORD, Elizabeth J., A history of childhood and disability, New York, Teachers College Press - Coloumbia University, 1996. Si rimanda inoltre a un confronto con ALBRECHT, Gary L., SEELMAN, Katherine, BURY, Michael (eds.), Handbook of Disability Studies, Thousand Oaks - London - New Delhi, Sage Publications, 2001, caratterizzato da un approccio interdisciplinare. 
tantomeno da un punto di vista storiografico. Nel considerare gli studi più significativi, si possono citare quelli di Brent e Kirsten M. Lovelock, di Small e Darcy, Corvo, e, in linea più generale e sotto una prospettiva più sfaccettata, i numerosi contributi nel volume Mind and body spaces curato da Butler e Parr. Opere in cui prevale tuttavia l'ottica sociologica e antropologica ${ }^{6}$.

Il presente articolo si pone l'obiettivo di delineare un quadro di carattere storiografico che sia dedicato alla situazione italiana, considerando l'evolversi dell'inclusione delle persone disabili in ambito turistico nella seconda metà del Novecento: un evolversi tutt'altro che lineare e che, se da una parte ha consentito di raggiungere importanti obiettivi, dall'altra non può nemmeno oggi dirsi del tutto soddisfacente.

A tal fine, in questa sede si intende fare riferimento ad alcune tipologie di turismo che hanno consentito a molte persone disabili di poter usufruire in Italia di attività ricreative lontane da casa per periodi continuati, in epoche nelle quali le barriere architettoniche erano per loro invalicabili. Ci si riferisce ad alcune esperienze riconducibili alle categorie dei viaggi religiosi, del turismo di cura della persona e del turismo nelle colonie estive. In un simile contesto, era impensabile che gli individui disabili si muovessero da soli ed era necessario che si appoggiassero alla famiglia o si affidassero ad appositi enti. Di conseguenza, il supporto alle persone disabili e il loro coinvolgimento in occasioni ricreative, culturali o che potessero essere ascritte all'esperienza turistica era un fatto gestito nella maggior parte dei casi privatamente, in Italia come all'estero.

Innanzitutto, si ripercorrerà l'evoluzione della normativa italiana in fatto di disabilità e inclusione, con un particolare riguardo a eventuali collegamenti con l'ambito turistico. Importante in questa parte sarà l'analisi dei punti di rottura tra la situazione delineata dalle normative dei tardi anni Ottanta e degli anni Novanta, le più attente all'inclusione sociale e culturale delle persone disabili, e la situazione precedente. In seguito, poiché nella letteratura storiografica italiana non è possibile rinvenire una precisa classificazione delle forme di turismo che hanno coinvolto nei decenni i viaggiatori disabili, verrà proposto un quadro storiografico che funga da riferimento teorico ed empirico per la periodizzazione del nucleo tematico in analisi. Si partirà proprio dalle tipologie di turismo precedentemente citate: viaggi religiosi, soggiorni finalizzati alla cura del corpo, colonie.

Se fino a questo punto verrà considerata la disabilità in senso generale - come insieme di specifiche ulteriori disabilità fisiche, psichiche e sensoriali - nella seconda parte del contributo sarà proposto un case study che avrà come protagoniste persone con una disabilità più definita, quella visiva, nonostante l'ampio spettro di situazioni individuali. Tale case study sarà infatti

\footnotetext{
6 LOVELOCK, Brent, LOVELOCK, Kirsten M., The Ethics of Tourism. Critical and applied perspectives, Abingdon, Routledge, 2013; SMALL, Jennie, DARCY, Simon, op. cit.; BUTLER, Ruth, PARR, Hester (eds.), Mind and body spaces: geographies of illness, impairment and disability, London, Routledge, 1999; CORVO, Paolo, Turisti e felici? Il turismo tra benessere e fragilità, Milano, Vita e Pensiero, 2007.
} 
relativo alla gestione e alla proposta ricreativa nelle colonie estive - balneari e montane - alle quali si appoggiava l'Istituto dei Ciechi di Milano.

\section{La normativa italiana a proposito di disabilità e inclusione}

Fondamentale per l'integrazione della persona disabile nella società è stata l'approvazione della legge 5 febbraio 1992, n. 104, ossia la Legge-quadro per l'assistenza, l'integrazione sociale e i diritti delle persone handicappate ${ }^{7}$. In alcuni casi indirettamente in altri in maniera diretta, nella legge 104/92 è affrontata anche l'integrazione della persona disabile nell'attività turistica.

Il testo, all'ultima modifica registrata, specifica che la Repubblica Italiana garantisce i diritti di libertà e di autonomia della persona handicappata, promuovendone l'integrazione nella famiglia, nella scuola, nel lavoro e nella società. Nel compiere questo, la Repubblica "previene e rimuove» qualsiasi condizione invalidante che impedisca alla persona disabile di raggiungere la massima autonomia possibile e la partecipazione alla vita della collettività, anche predisponendo degli interventi che abbiano lo scopo di scongiurare emarginazione ed esclusione sociale dell'individuo disabile. Più avanti nel testo, la stessa legge-quadro elenca tra i mezzi per realizzare l'inserimento e l'integrazione sociale della persona disabile interventi che servano «ad eliminare o superare le barriere fisiche e architettoniche che ostacolano i movimenti nei luoghi pubblici o aperti al pubblico», ma anche un "adeguamento delle attrezzature e del personale dei servizi educativi, sportivi, di tempo libero e sociali».

Ma è l'art. 23 che fa diretto riferimento alla sfera turistica: «Rimozione di ostacoli per l'esercizio di attività sportive, turistiche e ricreative». In particolar modo, nel terzo comma viene affrontata la questione degli impianti di balneazione e loro rinnovi, «subordinati alla visitabilità degli impianti [...] e all'effettiva possibilità di accesso al mare delle persone handicappate», con specifico riferimento al decreto del Ministero dei lavori pubblici 14 giugno 1989, n. 236 (Prescrizioni tecniche necessarie a garantire l'accessibilità, l'adattabilità e la visitabilità degli edifici privati e di edilizia residenziale pubblica sovvenzionata e agevolata, ai fini del superamento e dell'eliminazione delle barriere architettoniche) che contiene norme di attuazione della legge 9 gennaio 1989, n. 13 (Disposizioni per favorire il superamento e l'eliminazione delle barriere architettoniche negli edifici privati).

\footnotetext{
7 Gazzetta Ufficiale della Repubblica Italiana (d'ora in avanti GU), serie generale, 17 febbraio 1992, n. 39, suppl. ordinario n. 30. Come precisato dal sito HandyLex.org, curato dal Centro per la documentazione legislativa dello UILDM, il testo della legge 104/92 è stato modificato nei successivi venticinque anni fino al decreto legislativo 13 aprile 2017, n. 66, URL: < http://www.handylex.org/stato/l050292.shtml > [consultato il 14 novembre 2018].
} 
Proprio la legge $13 / 89$ e, nell'arco di pochi mesi, il decreto $236 / 89^{8}$ hanno dato un contributo molto importante alla normativa che riguarda la fruibilità da parte delle persone disabili dell'ambiente che li circonda e alla loro possibilità di muoversi sia all'interno di costruzioni sia in spazi esterni, peraltro definendo i criteri di progettazione che riguardano entrambe le tipologie. Subito all'art. 2 del decreto ministeriale si specifica che le barriere architettoniche sono quegli ostacoli fisici che costituiscono un disagio per la mobilità di chiunque, ma soprattutto per coloro che hanno una capacità motoria ridotta o impedita. Vi è un particolare riguardo per gli individui con disabilità visiva, poiché si sottolinea come tra le barriere incriminate rientri anche «la mancanza di accorgimenti e segnalazioni che permettono l'orientamento e la riconoscibilità dei luoghi e delle fonti di pericolo per chiunque e in particolare per i non vedenti, per gli ipovedenti e per i sordi». Nel 1996, a coronamento di quanto iniziato nel 1989, si giunge al decreto n. 503 del Presidente della Repubblica, ovvero il Regolamento recante norme per l'eliminazione delle barriere architettoniche negli edifici, spazi e servizi pubblici'. Queste disposizioni - ricordando anche l'imprescindibile legge-quadro 104/92 - hanno cambiato radicalmente il modo di percepire la disabilità: non più come una problematica individuale, bensì come una questione alla quale si deve interessare e deve provvedere l'intera società. Inoltre, è stato notevole il loro impatto sul mondo del turismo e sull'adeguamento di infrastrutture e servizi in modo che siano fruibili anche dai turisti disabili: anche se questo può essere affermato soltanto dal punto di vista teorico, perché a livello concreto non sempre è rispettata - o rispettata del tutto - la normativa vigente ${ }^{10}$.

Molto diversa la situazione precedente ai vari di fine anni Ottanta. Nonostante la Costituzione della Repubblica Italiana specifichi che tutti i cittadini sono uguali, con pari dignità e libertà, e si impegni a rimuovere gli ostacoli che impediscono il concretizzarsi di questi principi ${ }^{11}$, per decenni l'integrazione delle persone disabili nei diversi ambiti sociali procedette a singhiozzi, in maniera disorganica e con molte contraddizioni, comunque trascurandole in qualità di turisti e privilegiando la problematica dell'integrazione in rapporto ad ambiti più necessari, come il lavoro o l'istruzione.

\footnotetext{
$8 \mathrm{GU}$, serie generale, 23 giugno 1989 , n. 145, suppl. ordinario n. 47: il testo del decreto ministeriale è alle pp. 5-50, mentre il testo aggiornato della legge 13/89 si trova alle pp. 51-55.

$9 \mathrm{GU}$, serie generale, 27 settembre 1986, n. 227, suppl. ordinario n. 160. Cfr. Corvo, Turisti e felici?, cit., pp. 8687.

${ }^{10}$ Importante in questo ambito è il concetto di un «universal design» che predisponga ambienti privi di barriere e accessibili a persone anziane e a disabili: nel caso specifico dell'industria turistica, strutture alla cui base si trovino principi universali di progettazione che tengano conto dei fruitori con situazioni differenti da quelle considerate «nella norma». In merito, si rimanda a: COLE, Stroma, MORGAN, Nigel, Introduction: tourism and inequalities, in IID. (eds.), Tourism and inequality. Problems and prospects, cit., pp. xvxxiii, in particolare pp. xvii-xviii; BUTLER, Ruth, PARR, Hester, New geographies of illness, impairment and disability, in IID. (eds.), Mind and body spaces: geographies of illness, impairment and disability, cit., pp. 1-25; IMRIE, Rob, The body, disability and Le Corbusier's conception of the radiant environment, in BUTLER, Ruth, PARR, Hester (eds.), Mind and body spaces: geographies of illness, impairment and disability, cit., pp. 25-44, in particolare pp. 39-40.

${ }^{11}$ Si tratta dell'art. 2 e soprattutto dell'art. 3 nei principi fondamentali.
} 
Da ricordare in questa sede che, prima della legge 104/92, il principale punto di riferimento per le questioni di integrazione delle persone disabili fu la legge 30 marzo 1971, n. 118, la quale convertì il decreto-legge 30 gennaio 1971, n. 5 e perlomeno incentivò l'ingresso di disabili psichici e soprattutto fisici nel resto della società - scuola, università, ambienti di lavoro - contribuendo a ridurre forme di pregiudizio basate sulla mera ignoranza nei confronti di quello che era vissuto come un vero e proprio mondo a parte. È da tenere conto che, pur marginalmente, l'art. 27 prendeva in considerazione le problematiche causate dalle barriere architettoniche e le limitazioni sui mezzi di trasporto pubblici ${ }^{12}$.

\section{Turismo per persone disabili nel corso del Novecento italiano}

Quando non esisteva una normativa italiana che si occupasse in maniera adeguata e diretta dell'integrazione dell'individuo disabile nell'offerta turistica, la soluzione era andare in vacanza con la propria famiglia oppure affidarsi ad associazioni, enti, istituti civili o religiosi che organizzavano forme di villeggiatura riconducibili alle tre tipologie di viaggi e soggiorni a cui si è fatto cenno in apertura: viaggi religiosi, turismo di cura della persona, turismo nelle colonie estive. Poiché in questo articolo si considera lo scenario dell'Italia nel Novecento, è opportuno tracciare un quadro di sviluppo del fenomeno turistico nella penisola in cui inserire le tre tipologie in questione.

Gli studi che in questa sede si sono presi come riferimento e da cui parte l'interpretazione finalizzata a proporre il successivo impianto teorico ed empirico sono essenzialmente quelli di Patrizia Battilani, di Annunziata Berrino e di Giuseppe Rocca: si ritiene che siano i più aggiornati, completi e adatti a fornire, con la giusta lettura critica, il background da cui partire per trattare il nucleo argomentativo «turismo-disabilità-inclusione» e proporre un'ulteriore classificazione della storia del turismo in rapporto alle persone disabili, che a oggi in Italia manca ${ }^{13}$.

Sono cinque le epoche in cui è possibile scandire lo sviluppo del fenomeno turistico: prototurismo, Grand Tour, turismo moderno, turismo di massa e turismo «postmoderno». A interessare il Novecento furono le ultime tre fasi, a partire dal turismo moderno, che ebbe origine nel secondo Ottocento e durò fino agli inizi del secolo successivo ${ }^{14}$. Occuparsi di storia del turismo

\footnotetext{
${ }_{12} \mathrm{GU}$, serie generale, 2 febbraio 1971, n. 82, pp. 1955-1960: Conversione in legge del decreto-legge 30 gennaio 1971, n. 5, e nuove norme in favore dei mutilati ed invalidi civili. Per l'art. 27, ivi, p. 1959.

13 BATTILANI, Patrizia, Vacanze di pochi vacanze di tutti. L'evoluzione del turismo europeo, Bologna, Il Mulino, 2009; BERRINO, Annunziata, Storia del turismo in Italia, Bologna, Il Mulino, 2011; ROCCA, Giuseppe, Dal prototurismo al turismo globale. Momenti, percorsi di ricerca, casi di studio, Torino, Giappichelli, 2013. Si propone anche un confronto con: ECHTNER, Charlotte M., JAMAL, Tazim B., «The disciplinary dilemma of tourism studies», in Annals of Tourism Research, 24, 4, 1997, pp. 868-883; MAFFI, Luciano, Turismo dell'Ottocento. I viaggi in Italia di un prete pavese, Milano, Il Cisalpino, 2015, pp. 24-29.

14 Tra gli italiani il turismo moderno si diffuse a partire dal periodo postunitario, con il rafforzamento della
} 
in relazione alla disabilità significa quindi porsi in relazione a delle categorie storiografiche relative al fenomeno turistico già esistenti, a fasi di sviluppo del fenomeno anche rapide e complesse che si sono verificate nel corso dell'ultimo secolo ${ }^{15}$. Certo è che le classificazioni adatte a una domanda turistica più generica sono da riconsiderare quando si entra nello specifico del turismo usufruibile da individui disabili e, nel caso dell'Italia, specialmente prima degli interventi legislativi degli anni Settanta e poi della legge 104/92. D'altro canto i turisti disabili condividono con tutti gli altri turisti aspettative, desideri, passioni, che nel corso del tempo il legislatore ha cercato di assecondare favorendo l'integrazione e riducendo gli ostacoli architettonici.

Innanzitutto si prenda in esame il turismo religioso, che dal punto di vista storico ha costituito una delle prime tipologie di turismo, presente già nelle società antiche. I pellegrinaggi erano e sono il modo attraverso cui l'uomo si relaziona con il divino: tramite il pellegrinaggio l'animo umano si apre all'Assoluto, segue un percorso interiore proiettato alla meditazione, al raccoglimento, al pentimento, ma anche a un desiderio di conoscenza. Come nell'antichità il pellegrinaggio poteva essere devozionale, espiatorio, mirato alla ricerca di una guarigione miracolosa, anche oggi i fedeli si recano nei luoghi sacri per rimettere in mani sante e divine preghiere, ansie e intenzioni, ricercando purificazione spirituale. Dal canto suo, il pellegrinaggio cristiano iniziò a diffondersi soltanto durante l'Alto Medioevo ${ }^{16}$. Il cristiano di allora si riconosceva perfettamente nella figura del pellegrino. In un'epoca di forte sensibilità religiosa come il Medioevo, il pellegrinaggio e le sue dure prove fisiche non rispondevano solo a una volontà di espiazione dei peccati, ma miravano a realizzare una conoscenza più personale e diretta della fede.

nuova classe borghese. Il turismo di massa, invece, in Italia nacque nei primi anni Sessanta del Novecento, durante il miracolo economico, e si sviluppò nei decenni successivi: vero e proprio bene di consumo, era alla portata di tutti i ceti sociali e comprendeva soggiorni all'estero per motivi di studio oppure di turismo sportivo. Infine, a partire dagli anni Novanta, ecco il turismo postmoderno o «esperienziale», quello che stiamo ancora vivendo, in cui non sono più le mete a fare la differenza, ma le esperienze che il turista può vivere. Quello che accomuna i turisti non è più soltanto la capacità di spesa o la classe sociale di provenienza, ma il tipo di esperienza desiderata. In merito alla situazione italiana, si veda: BATTILANI, Patrizia, op. cit., pp. 224-275. Utile un confronto con il quadro internazionale tracciato in ROCCA, Giuseppe, op. cit., pp. 31-94. Si precisa infine che, per la situazione italiana nei decenni più recenti, Berrino pone l'accento su una visione «olistica» del turismo, incentrata sulla ricerca del benessere totale legato all'attività sportiva, alla buona alimentazione e alla tranquillità dell'ambiente, in un contesto che valorizza maggiormente «forme di ospitalità caratteristiche». In merito si rimanda a: BERRINO, Annunziata, Storia del turismo in Italia, cit., pp. 288-298.

15 La storiografia contemporanea si è soffermata sull'analisi dei fattori che hanno stimolato la domanda di turismo: il tempo libero e il reddito, che devono coesistere. Il primo è una precondizione, perché lo sviluppo del fenomeno turistico in tutta la sua storia si lega al tempo libero, le cui modalità di impiego sono mutate sul lungo periodo. Il reddito è invece l'elemento economico principale che condiziona i consumi turistici: il raggiungimento di una soglia di reddito sufficiente per permettersi di fare turismo è stata una conquista per categorie sociali prima escluse (BATTILANI, Patrizia, op. cit., pp. 23-32). Infine, c'è un terzo fattore che condiziona e stimola la domanda di turismo, meno misurabile e soggettivo: il desiderio e la passione per il viaggio. Tale aspetto è fondamentale perché determina la scelta di spendere il proprio tempo libero e il proprio reddito in attività turistiche. Si veda MAFFI, Luciano, op. cit., pp. 9-13.

${ }^{16}$ BATTILANI, Patrizia, op. cit., pp. 65-86. 
Ritornando alla contemporaneità, nel Novecento cristiano i santuari delle apparizioni mariane hanno avuto un ruolo centrale nei viaggi e nei soggiorni delle persone disabili. Si pensi a uno dei luoghi più famosi in questo ambito: Lourdes, dove la Madonna è deputata alla guarigione del corpo, il che ha sempre convogliato flussi turistici massicci e in continua crescita, tanto che oggi questa località ha raggiunto oltre 6 milioni di visite l'anno ${ }^{17}$. Si tratta però di un nuovo tipo di turismo religioso: non si cammina più, si viaggia in treno, pullman e aereo. In genere, questi pellegrinaggi durano pochi giorni e spesso sono organizzati da associazioni religiose che hanno come scopo questo fine: in Italia, per esempio, ci sono l'UNITALSI (Unione Nazionale Italiana Trasporto Ammalati a Lourdes e Santuari Internazionali), attiva dai primi del Novecento, e l'OFTAL (Opera Federativa Trasporto Ammalati a Lourdes), nata negli anni Trenta.

Un altro tipo di viaggio-soggiorno che nel Novecento è stato importante per gli individui disabili è quello relativo alla cura del corpo, in località termali o altri luoghi di cura ${ }^{18}$. Il turismo termale usufruisce di acque sorgenti, vapori e fanghi che possiedono proprietà curative per i bisogni più disparati. Da un lato c'è il benessere, un concetto antico con moltissime sfaccettature, che arriva a coinvolgere corpo, mente e spirito. Dall'altro lato si trova la salute, dei cui aspetti fu maturata una conoscenza più approfondita a partire dal Settecento e dall'Ottocento, con l'evoluzione della medicina, l'affermarsi del metodo sperimentale, lo sviluppo della chimica moderna che rese possibili le prime indagini sulla composizione delle acque minerali. Le località termali sin dalle loro origini, inoltre, con la loro tipica organizzazione - stabilimento termale, hotel, parco e attività di svago - hanno spesso favorito le persone disabili dal punto di vista dell'accessibilità, della sicurezza e della tranquillità ${ }^{19}$.

Infine, il tipo di soggiorno correlato al case study: le colonie estive. Sorte già nel corso dell'Ottocento, ebbero come scopo principale quello di inviare gruppi di bambini e ragazzi alle cure climatiche per scopi di salute sia in montagna sia al mare. In riferimento alla specifica situazione italiana, erano patrocinate soprattutto da benefattori e da Comuni e, secondo la legge delle opere pie (legge 17 luglio 1890, n. 6972), ottennero la qualifica di enti autonomi. Inizialmente, ebbero una valenza ospedaliera di supporto alle cure per giovani con tubercolosi

\footnotetext{
17 Si veda: LAVARINI, Roberto, Il pellegrinaggio cristiano: dalle origini al turismo religioso del XX secolo, Genova, Marietti, 1997.

${ }^{18}$ Fanno riferimento al turismo di cura i seguenti articoli pubblicati in Annale di Storia del Turismo: BERRINO, Annunziata, «La nascita delle Aziende autonome e le politiche di sviluppo locale in Italia tra le due guerre», in Annale di Storia del Turismo, V, 2004, pp. 33-54; KAWAMURA, Ewa, «Il soggiorno dei tisici inglesi negli alberghi italiani e svizzeri tra Ottocento e Novecento", in Annale di Storia del Turismo, VI, 2005, pp. 9-34.

19 Per quanto concerne il turismo termale e le strutture delle località termali si rimanda ai seguenti studi: ROCCA, Giuseppe, Dal prototurismo al turismo globale, cit., pp. 139-182; ID. (a cura di), «Dai luoghi termali ai poli e sistemi locali di turismo integrato", in Geotema, XXVIII, 1/2006, pp. 32-33; BOSSAGLIA, Rossana, Stile e struttura delle città termali. Lombardia, Piemonte, Valle d'Aosta, Bergamo, Milano, Banca Provinciale Lombarda, 1984; ID., Stile e struttura delle città termali. Veneto, Trentino-Alto Adige, Friuli-Venezia Giulia, Emilia-Romagna, Toscana, Campania, Sicilia, Milano, Banca Provinciale Lombarda, 1985.
} 
ossea o articolare, o più in generale con gracilità accentuata o disabilità, con la gestione delle strutture spesso affidata a religiosi.

Lo sviluppo maggiore di questi soggiorni fu nel corso del Novecento, quando le colonie temporanee vennero destinate anche ai figli dei combattenti. Inoltre, l'azione di paternalismo aziendale nelle grandi imprese e il supporto ai lavoratori da parte di sindacati o enti religiosi sostennero lo sviluppo di queste forma di vacanze ${ }^{20}$. Non a caso, in Italia il loro incremento novecentesco si correlò con le vicende politiche ed economiche del Paese: nel periodo fascista furono potenziate e usate come luoghi di propaganda e di formazione della gioventù. Nel 1927 i bambini ospitati furono 54.000 , mentre nel 1938 si arrivò a 772.000 in colonie sparse su tutto il livello nazionale, con una concentrazione sul litorale toscano e romagnolo ${ }^{21}$.

Nel secondo dopoguerra recuperarono il ruolo di supporto medico, cui si aggiunse una valenza sociale e ricreativa, permettendo ai giovani di svolgere un periodo di vacanza al mare o in montagna anche quando all'intera famiglia non era possibile andare in vacanza. In seguito, a partire dagli anni Settanta, ci fu un lento declino le cui cause sono da ricercare nell'aumento di salari e redditi delle famiglie - i cui componenti potevano così recarsi assieme in vacanza - e ancora nei tagli alla spesa sociale da parte di enti pubblici e aziende.

\section{L’Istituto dei Ciechi di Milano e il suo archivio storico}

L'Istituto dei Ciechi di Milano nacque nel 1839 su proposta di Michele Barozzi e approvazione dell'Imperial Regio Governo austriaco, entrando in funzione nel luglio dell'anno successivo. Dopo le prime sedi provvisorie, nel 1892 venne definitivamente installato nel palazzo Barozzi, costruito appositamente in via Vivaio su progetto dell'architetto Giuseppe Pirovano; in seguito, nel corso del primo decennio del Novecento, fu organizzato e inaugurato dall'allora direttore Luigi Vitali un asilo per trenta bambini nella fascia di età quattro-otto anni: situato nei pressi dell'Istituto, era però autonomo. Come precisa Maria Canella, nell'asilo si applicava il metodo Montessori incentrato sull'educazione sensoriale, adeguato agli specifici bisogni dell'utenza ${ }^{22}$. Un marchio di

\footnotetext{
${ }^{20}$ Sulle abitudini di vita in colonia: MUCELLI, Elena, Colonie di vacanza italiane degli anni '30: architetture per l'educazione del corpo e dello spirito, Firenze, Alinea, 2009, pp. 23-44. Sul ruolo sociale delle colonie: CARERA, Aldo, Paternalismo, responsabilità sociale e forme di partecipazione nel secondo dopoguerra: le opere sociali per l'infanzia, in MINESSO, Michela (a cura di), Welfare e minori. L'Italia nel contesto europeo del Novecento, Milano, FrancoAngeli, 2011, pp. 321-349. Sulle colonie durante il periodo fascista: DE MARTINO, Stefano, WALL, Alex (eds.), Cities of Childhood. Italian Colonie of the 1930s, London, Architectural Association, 1988.

${ }^{21}$ Queste informazioni sono tratte dalla seguente inchiesta: BERTUCCIOLI, Sara, «Dai fasti degli anni '30 al declino dei '70 le colonie marine tra cura e vacanze», in la Repubblica.it, URL:

<http://inchieste.repubblica.it/it/repubblica/rep-it/2012/08/20/news/dai_fasti_degli_anni_30_al_declino_ dei_70_le_colonie_marine_tra_cura_e_propaganda-40008066/ > [consultato il 2 ottobre 2018].

${ }^{22}$ Sulla storia dell'Istituto fino al 1945 si rimanda a: CANELLA, Maria, Profilo storico dell'Istituto dei Ciechi dagli esordi al 1945, in BASCAPÈ, Marco G., CANELLA, Maria, REBORA, Sergio (a cura di), Luce su luce. L'impegno della
} 
notevole modernità pedagogica ed educativa che si sarebbe rispecchiato anche nella pianificazione delle colonie estive del secondo Novecento studiate nel presente articolo.

Lo studio che ha come oggetto i soggiorni estivi dei ragazzi e delle ragazze dell'Istituto dei Ciechi di Milano si fonda sullo spoglio e sull'analisi di materiale conservato presso l'archivio storico dell'Istituto medesimo ${ }^{23}$. I documenti di carattere amministrativo specifici per le colonie estive sono disponibili per gli anni dal 1955 al 1975 e consentono di indagare numerosi fattori relativi alle vacanze degli studenti24. A essi vanno aggiunte alcune carte del consiglio d'amministrazione, che permettono di tracciare un quadro più completo del valore delle iniziative in questione ${ }^{25}$.

In primo luogo, si tratta di aspetti legati alla destinazione, sempre correlata al supporto psicofisico della persona disabile nella sua fanciullezza e adolescenza. Inoltre, sono presenti informazioni circa la selezione della meta di soggiorno, con almeno una doppia offerta - in alcuni anni, addirittura più variegata - per i ragazzi e le ragazze disabili, da indirizzare verso una colonia di mare o verso una di montagna che non sono sempre le stesse nell'arco cronologico considerato. Ulteriori dati sono relativi ai costi delle vacanze, sulla quota del viaggio e del soggiorno, sul pagamento del personale di assistenza, sul periodo della vacanza. Altre informazioni sono quelle invece riferite ai fruitori di questo servizio, con nomi, età e Comune di residenza dei ragazzi. Inoltre, ci sono indicazioni sul corredo che devono portare per il soggiorno, sull'alimentazione e sulle attività giornaliere. Talvolta, specie dalla rendicontazione economica aggiuntiva scritta dal personale di assistenza, conoscono attività aggiuntive, come cinema, luna-park, spettacoli di burattini, e alimenti extra, come gelati.

Le fonti analizzate ci informano del fatto che l'Istituto organizzava e gestiva le vacanze, quindi si metteva a servizio dei propri fruitori anche al di fuori del periodo scolastico, pur non sostenendone le spese per la quota di soggiorno, se non in alcuni casi relativi a studenti particolarmente bisognosi. Grazie allo studio di queste carte è stata possibile l'interessante

solidarietà dalla carità alla scienza, Milano, Istituto dei Ciechi, 2003, pp. 18-53. Sull'installazione dell'Istituto in via Vivaio e sulla modernità dell'asilo il rimando è alle pp. 38-39 e relative note. Nel 1924 il metodo Montessori era già applicato presso l'asilo, come si può leggere in L'Istituto dei ciechi di Milano. L'opera, $i$ risultati, i mezzi 1840-1924, Varese, Tipografia Varesina, 1924, pp. 13-15; in particolare, a p. 15 si legge: «Viene applicato, nei limiti imposti dalla cecità, il metodo di educazione sensoriale ideato dalla dottoressa Montessori, e già applicato in America ai ciechi». Per una genesi del metodo elaborato da Maria Montessori (1870-1952) e per la sua ricezione su entrambe le coste dell'Atlantico, si rimanda a: KRAMER, Rita, Maria Montessori. A biography, Chicago, University of Chicago Press, 1976.

${ }^{23}$ Si ringrazia Enrica Panzeri, responsabile dell'Archivio storico dell'Istituto dei ciechi, per la gentilezza, la disponibilità e la precisione della sua assistenza. Un ringraziamento va anche a Melissa Tondi, conservatore museale della raccolta Louis Braille dell'Istituto. Per una storia dell'archivio e una sua descrizione si rimanda a PANZERI, Enrica, L'archivio storico, in BASCAPÈ, Marco G., CANELLA, Maria, REBORA, Sergio (a cura di), op. cit., pp. 284-291.

24 Archivio storico Istituto dei Ciechi di Milano (d'ora in avanti ASICMi), Assistenza e Istruzione (ex Beneficenza), Colonie estive, bb. 1-2, fascc. 1-21.

${ }^{25}$ ASICMi, Assistenza e Istruzione (ex Beneficenza), Rettori, b. 4, fasc. 25. 
ricostruzione del profilo degli attori sociali che sostenevano tali attività, come si avrà modo di spiegare nei particolari tra qualche riga. In questa sede, si precisa che dai documenti di carattere amministrativo emerge anche un ulteriore tipo di fonte, di grande interesse pedagogico oltre che storiografico, utile in questo tipo di analisi che unisce destinazione e destinatario: si tratta di alcune relazioni del personale che accompagnava i giovani ciechi, da cui emergono le complessità di gestione e le riflessioni sulle strutture ospitanti.

Presso l'Istituto dei Ciechi sono conservati anche i numeri della rivista «Alba Serena», curata dall'ente medesimo ${ }^{26}$, una fonte particolarmente preziosa perché consente di integrare le informazioni amministrative con gli articoli pubblicati che riportano descrizioni e riflessioni sui periodi vacanzieri, oltre alle impressioni dei ragazzi sulle colonie e sulle attività ricreative svolte.

Si tenga infine conto che gli anni Cinquanta, dai quali parte l'analisi delle colonie estive, furono sin da subito scenario di importanti innovazioni all'interno dell'Istituto. Per esempio, all'inizio del $1951 \mathrm{fu}$ approvato dal Presidente della Repubblica Einaudi il nuovo statuto dell'ente; quest'ultimo risultava così suddiviso in tre sezioni, ovvero l'asilo per i bambini tra i quattro e i sei anni, il collegio convitto dedicato ai successivi otto anni per l'istruzione obbligatoria elementare e l'introduzione alle professioni, la casa famiglia per donne cieche adulte - già sorta con le modifiche del 1925 - che versavano in condizioni di indigenza o che comunque non avevano supporto familiare. Rientravano in questo clima di cambiamento anche le modifiche relative al recupero delle risorse: al di là di rendite, lasciti e beneficenza varia, pagano ricovero, formazione e assistenza soprattutto le amministrazioni provinciali, vari enti pubblici e privati e le famiglie di ospiti non indigenti. Inoltre, il nuovo consiglio d'amministrazione era formato da sette membri, di cui due e il presidente indicati dal Comune di Milano, uno dall'amministrazione provinciale, due dai benefattori e uno dall'Unione Italiana dei Ciechi: tutti nomi che sarebbero comparsi anche nel finanziamento delle colonie estive. Il primo consiglio d'amministrazione del nuovo periodo fu attivato dall'estate dell'anno successivo ${ }^{27}$.

In seguito, tra il 1953 e il 1956, ebbe luogo una serie di polemiche tra la Federazione Nazionale delle Istituzioni pro Ciechi e alcuni istituti che culminò quando la prima presentò un progetto di legge «teso a far inquadrare nei ruoli statali i direttori degli istituti per ciechi con annesse scuole elementari di Stato». I rappresentanti di Milano, Padova e Assisi consideravano dannoso per la gestione degli istituti lo «svincolo di ogni legame giuridico-disciplinare del capo della comunità

${ }^{26}$ Il varo di questa e di altre riviste rientrò, già nella prima metà del Novecento, in una serie di iniziative dell'Istituto mirate a far conoscere le proprie attività e a creare un ponte con il resto della società. In merito si rimanda a FUMI, Gianpiero, Le molte vie del finanziamento privato del bene pubblico. Milano tra Ottocento $e$ Novecento, in TOGNETTI BORDOGNA, Mara, SIRONI, Vittorio A., Milano capitale del bene comune, Milano, FrancoAngeli, 2013, pp. 77-111, nello specifico pp. 81-83.

27 M. Granata, Le vicende dell'Istituto dei Ciechi dal secondo dopoguerra a oggi, in BASCAPÈ, Marco G., CANELLA, Maria, REBORA, Sergio (a cura di), Luce su luce, cit., pp. 56-79; per il rinnovo dello statuto si vedano le pp. 6163. 
interna dall'autorità del consiglio d'amministrazione», pertanto protestarono agguerriti. Quando la legge venne presentata all'inizio del 1956, l'Istituto di Milano fece ricorso al Consiglio di Stato e se ne andò dalla Federazione: vi sarebbe tornato solo nel 1960. A metà anni Sessanta, invece, alcuni istituti della Federazione espresso il proprio dissenso nei confronti di un altro progetto di legge, questa volta caldeggiato dall'Unione Italia dei Ciechi: era mirato alla statalizzazione degli istituti o comunque a parte di essi e quello di Milano non lesinò sulle proteste. Inevitabilmente, però, si giunse a una convenzione tra l'Istituto e il Conservatorio cittadino con cui il primo dismise la scuola musicale a vantaggio del secondo. Infine, nel decennio successivo, subentrò la questione legata alla legge 11 maggio 1976, n. 360, che a Milano avrebbe svuotato il convitto dell'Istituto; si riuscì però a conservare il servizio scolastico, comunque privato della scuola elementare speciale per ciechi ${ }^{28}$.

Tracciato questo quadro istituzionale - in cui è già possibile notare il ruolo attivo e sempre aggiornato dell'Istituto - e date le coordinate metodologiche per l'indagine, si passerà ora al case study delle colonie.

\section{L'attività ricreativa nelle colonie estive dell'Istituto}

In prima istanza, è da ricordare ancora una volta come l'obiettivo delle colonie estive fosse quello di favorire il benessere psicofisico dei ragazzi dell'Istituto. Un responsabile sanitario, infatti, visitava previamente i giovani, indicando le mete e le attività più adeguate alla loro condizione; l'invio in colonia era legato quindi al bisogno dei ragazzi ciechi di «cure fisiche» che potessero influenzare positivamente il «futuro rendimento scolastico». A tal proposito, era assegnata una valenza sociale e pratica a questo genere di vacanza, poiché i benefici che ogni ragazzo traeva da un periodo al mare o in montagna con i compagni giovava anche all'intero Istituto e ai finanziatori, come le amministrazioni provinciali, «in quanto accelerando lo sviluppo fisico e psichico del ragazzo, spesso si evita che il medesimo abbia a ripetere una classe e ridurre così di qualche anno l'assistenza scolastica» ${ }^{29}$. Il ragazzo era quindi considerato come un futuro membro attivo della società, con il potenziale di contribuire appieno alla collettività e di partecipare alla vita lavorativa.

L'amministrazione dell'Istituto, inoltre, dedicava una particolare attenzione alla scelta delle mete di colonia. Questa cura era motivata sia dagli obiettivi di supporto psicofisico sia da motivi di praticità. Ogni anno la segreteria raccoglieva materiale informativo proveniente da enti che

\footnotetext{
${ }^{28}$ Ibidem, pp. 63-67 e 70-74, dove tutte queste vicende sono ricostruite in maniera più dettagliata e completa nella loro complessità.

29 ASICMi, Assistenza e Istruzione (ex Beneficenza), Colonie estive, b. 2, fasc. 8, l'Istituto dei Ciechi di Milano all'amministrazione provinciale, 21 aprile 1961.
} 
possedevano strutture di soggiorno in luoghi adatti alla vacanza, nei quali nella maggior parte dei casi i ragazzi erano organizzati secondo fasce di età ${ }^{\circ}$. Sul lungo periodo, è palese un rapporto fidelizzato con la federazione provinciale milanese dell'Associazione Nazionale Combattenti e Reduci, che metteva a disposizione le proprie strutture di San Mauro a Mare, in Romagna: erano queste la colonia Giovanni Pascoli e la colonia Cieli Azzurri; se la seconda era di recente costruzione, entrambe erano comunque spaziose e organizzate «con i criteri più moderni» ${ }^{11}$.

Dal 1960 in poi, accanto a San Mauro subentrarono alcune altre destinazioni marittime: la colonia a Gatteo a Mare, in provincia di Forlì, dell'ONARMO (Organizzazione nazionale assistenza religiosa morale operai); la colonia Santa Lucia all'Ardenza gestita dall'Istituto dei Ciechi di Livorno; la colonia Regina Mundi di Pinarella di Cervia; il soggiorno Leonessa a Villamarina di Cesenatico; la pensione San Basiano a Bellaria - Igea Marina; la colonia dell'ODA (Opera Diocesana Assistenza) a Marina di Massa. Invece, per quanto riguarda le sedi di montagna, un rapporto privilegiato era quello con l'Istituto Luigi Configliachi di Padova, che possedeva una colonia ad Asiago; tuttavia, anche in questo caso negli anni Sessanta furono proposte alcune destinazioni sostitutive, ovvero una struttura a Ciano d'Enza dell'Istituto dei Ciechi di Reggio Emilia, la colonia Narciso a Roncola San Bernardo e la pensione Stella Montis a Campitello di Fassa. Senza dimenticare l'adeguatezza delle strutture ospitanti, il tutto veniva organizzato all'insegna della maggiore inclusione possibile dei ragazzi ciechi: i quali, fatte le dovute eccezioni deducibili dagli elenchi sopra riportati, ero spesso ospitati al fianco di utenza vedente ${ }^{32}$. Per un elenco incrociato delle colonie estive, dei periodi di soggiorno interessati e dell'affluenza alle stesse, si rimanda alle tabb. 1A e 1B.

\footnotetext{
30 Alba Serena, XL, 5, dicembre 1962, p. 20, dove si legge che ad Asiago «la comunità, come di consueto, viene divisa in tre gruppi: il primo comprende i bambini sino ai 12 anni, cioè coloro che più abbisognano di premure. Il secondo comprende i giovanetti dai 12 ai 17 anni ed infine il terzo raccoglie i giovani superiori al $17^{\circ}$ anno». Altre volte, invece, questa divisione per fasce di età sembra effettuata a monte, in fase di organizzazione e comunque prima della partenza.

${ }^{31}$ ASICMi, Assistenza e Istruzione (ex Beneficenza), Colonie estive, b. 1, fasc. 1, brochure dell'Associazione per le colonie estive 1954; fasc. 3, elenco delle colonie estive organizzate dall'Associazione nel 1956. ${ }^{32}$ La successione delle mete è ricavabile dallo spoglio dei documenti in bb. 1-2, fascc. 1-21.
} 
Tab. 1A

\begin{tabular}{|c|c|c|c|c|c|}
\hline \multicolumn{6}{|c|}{ Colonie al mare } \\
\hline Anno & Meta & Totale ospiti & Maschi & Femmine & Periodo \\
\hline 1955 & San Mauro a Mare & 18 & 11 & 7 & 13 luglio - 11 agosto \\
\hline 1956 & San Mauro a Mare & 20 & 16 & 4 & 10 agosto -8 settembre \\
\hline 1957 & San Mauro a Mare & 17 & 10 & 7 & 21 agosto - 16 settembre \\
\hline 1958 & San Mauro a Mare & 11 & 4 & 7 & 22 agosto - 20 settembre \\
\hline 1959 & San Mauro a Mare & 12 & 6 & 6 & 21 agosto - 19 settembre \\
\hline 1960 & Gatteo a Mare & 25 & 15 & 10 & 10 luglio - 5 agosto \\
\hline 1961 & Ardenza & 14 & 5 & 9 & 1 agosto - 31 agosto \\
\hline 1962 & Ardenza & 27 & 16 & 11 & \\
\hline 1963 & Ardenza & & & & 1 agosto - 31 agosto \\
\hline 1964 & Ardenza & 41 & 19 & 22 & \\
\hline \multirow[t]{2}{*}{1965} & Ardenza & & & & \\
\hline & Gatteo a Mare & & & & \\
\hline \multirow[t]{2}{*}{1966} & Pinarella di Cervia & & & & \\
\hline & Villamarina di Cesenatico & 12 & & & 30 luglio - 20 agosto \\
\hline 1967 & Gatteo a Mare & 47 & & & \\
\hline \multirow[t]{2}{*}{1968} & Gatteo a Mare & 23 & & & \\
\hline & Villamarina di Cesenatico & 13 & & & \\
\hline 1969 & Gatteo a Mare & 38 & 18 & 20 & 25 luglio - 22 agosto \\
\hline 1970 & Gatteo a Mare & 13 & 8 & 5 & \\
\hline 1971 & Gatteo a Mare & 10 & 6 & 4 & 15 luglio - 10 agosto \\
\hline \multirow[t]{2}{*}{1972} & Bellaria - Igea Marina & 10 & & & \\
\hline & Gatteo a Mare & 17 & & & \\
\hline 1973 & Villamarina di Cesenatico & 15 & & & 18 luglio - 14 agosto \\
\hline 1974 & Marina di Massa & 15 & & & \\
\hline 1975 & Bellaria - Igea Marina & 22 & & & 4 luglio - 17 luglio \\
\hline
\end{tabular}

Tab. 1B

\begin{tabular}{|c|c|c|c|c|c|}
\hline \multicolumn{6}{|c|}{ Colonie in montagna } \\
\hline Anno & Meta & Totale ospiti & Maschi & Femmine & Periodo \\
\hline 1955 & Asiago & 8 & 6 & 2 & 11 agosto -6 settembre \\
\hline 1956 & Asiago & 6 & 4 & 2 & 18 giugno - 14 luglio \\
\hline 1957 & Asiago & 9 & 7 & 2 & 11 luglio - 6 agosto \\
\hline 1958 & Asiago & 11 & 6 & 5 & 9 agosto - 4 settembre \\
\hline 1959 & Asiago & 11 & 7 & 4 & 23 luglio - 18 agosto \\
\hline 1960 & Asiago & 10 & 5 & 5 & 11 giugno -7 luglio \\
\hline 1961 & Ciano d'Enza & 5 & 4 & 1 & \\
\hline 1962 & Asiago & 11 & 7 & 4 & \\
\hline 1963 & Ciano d'Enza & & & & 1 agosto - 31 agosto \\
\hline \multicolumn{6}{|l|}{1964} \\
\hline \multicolumn{6}{|l|}{1965} \\
\hline 1966 & Asiago & & & & \\
\hline \multirow[t]{2}{*}{1967} & Asiago & 13 & & & \\
\hline & Ciano d'Enza & 2 & & 2 & \\
\hline 1968 & Asiago & 14 & & & \\
\hline 1969 & Asiago & 14 & 11 & 3 & 27 giugno - 23 luglio \\
\hline \multicolumn{6}{|l|}{1970} \\
\hline \multicolumn{6}{|l|}{1971} \\
\hline \multirow[t]{2}{*}{1972} & Campitello di Fassa & 8 & & & \\
\hline & Roncola San Bernardo & 1 & & & \\
\hline 1973 & Campitello di Fassa & 10 & & & 15 luglio - 28 luglio \\
\hline 1974 & Campitello di Fassa & 5 & & & \\
\hline
\end{tabular}

Fonte: ASICMi, Assistenza e Istruzione (ex Beneficenza), Colonie estive, b. 1-2, fascc. 1-21; Rettori, b. 4, fasc. 25.

È giunta fino a noi anche una nutrita documentazione in merito alle attività ricreative nelle colonie, all'alimentazione cui si provvedeva e al corredo di vestiario che ciascuna famiglia doveva predisporre per il proprio ragazzo. Per quanto riguarda il primo di questi tre ambiti, sono 
fondamentali gli articoli di «Alba Serena», in cui si legge che al mare i ragazzini trascorrevano le giornate tra la frescura della pineta, i bagni a mare e la vita di spiaggia, abbronzandosi e costruendo castelli di sabbia. I gruppi era caratterizzati da una certa vivacità; per esempio, in un numero di «Alba Serena» del 1962 che tratteggia la vita di colonia all'Ardenza nell'estate del precedente 1961, il bagno a mare è definito come «il momento del batticuore per le assistenti che di notte (chissà, forse anche di giorno) sognavano una gran rete per tenerli [i ragazzini] a bada e poterli ripescare poi tutti sani e salvi»33. L'anno successivo ci sarebbero state attività musicali con il tamburello, esercizi ginnici, laute mangiate di lasagne e arrosti; nella stessa estate, ad Asiago era valorizzato il contatto con la natura alpina tra camminate, ore di riposo e attività di svago, con il venerdì dedicato alle gite più lunghe 34 .

Tornando alla colonia del mare, nell'estate 1964 l'UIC (Unione Italiana Ciechi) organizzò per i ragazzi all'Ardenza una gita al Santuario di Montenero. Nello stesso periodo alcune bambine fecero una gita a Pisa, mentre i maschi visitarono la nave da guerra Andrea Doria. In altre giornate, invece, furono organizzati spettacoli musicali e di cabaret35. Per l'estate del 1969 a Gatteo a Mare, furono pianificate gite in barca e pedalò, gite sugli scogli, gare di canto, gite per la cittadina e raccolta di conchiglie sulla spiaggia ${ }^{36}$.

Altrettanto interessanti gli eventuali report, diretti da assistenti alla direzione dell'Istituto, relativi alle attività estive e alla gestione delle colonie: uno particolarmente esteso è relativo proprio al soggiorno marittimo del 1969. Da questa fonte inedita si evince la complessità dell'organizzazione del periodo di vacanza; sono registrare anche alcune criticità al fine di migliorare l'offerta nelle successive esperienze educative. Le camerate dei maschi non erano molto comode e i giovani ospiti sentivano in maniera particolare «il disagio di un nuovo ambiente, molto diverso da quello in cui erano abituati a vivere», presumibilmente quello specializzato dell'Istituto di via Vivaio. Per quanto riguardava le bambine, si erano ritrovate in una camerata di dimensioni enormi «divisa da un mezzo muro da quella delle altre squadre»: circostanze che non consentivano il riposo per mancanza di tranquillità. Inoltre, i servizi igienici erano insufficienti rispetto al numero di ospiti. Tuttavia, superato l'iniziale periodo critico, il rapporto dei giovani ciechi con la geografia dell'ambiente migliorò gradualmente, consentendo loro di muoversi con maggiore sicurezza e riducendo il nervoso e l'agitazione, soprattutto nelle ore notturne ${ }^{37}$. La supervisione da parte del personale di accompagnamento dell'Istituto era

\footnotetext{
33 Alba Serena, XL, 4, luglio 1961 - maggio 1962, p. 30.

34 Alba Serena, dicembre 1962, cit., pp. 18-20.

35 Alba Serena, XLII, 2, novembre 1964, p. 26.

${ }^{36}$ Alba Serena, XLVIII, 1, maggio 1970, pp. 49-49.

37 ASICMi, Assistenza e Istruzione (ex Beneficenza), Colonie estive, b. 2, fasc. 15, report per la colonia marina 1969, foglio 1 recto.
} 
quindi attenta e critica, consapevole delle necessità sensoriali dei giovani che gli erano affidati, peraltro durante quell'estate in numero particolarmente elevato (tabb. 1A e 1B).

Nella colonia marina del 1969 ci furono inoltre alcuni problemi legati agli spazi ricreativi veri e propri, soprattutto il cortile, il quale aveva alcuni alberi considerati come un ostacolo al libero movimento di ciechi e cieche: anche questo un esempio delle barriere architettoniche di cui si è scritto all'inizio del contributo. Ancora una volta il personale di accompagnamento si occupò di trovare delle soluzioni che potessero garantire la sicurezza e al contempo la libertà degli assistiti: si isolò con delle panche uno spazio più sicuro in modo che ciechi e cieche non si facessero male e «non si mischiassero troppo con la moltitudine degli altri ragazzi». Una soluzione emblematica delle difficoltà che una persona disabile incontrava quotidianamente, anche in un contesto di svago e ralax come la vacanza: il personale, dovendo affrontare il bisogno di trovare un modo per allontanare ogni eventuale pericolo da assistiti e assistite dell'Istituto, non poté fare altro che procedere con una separazione parziale, che al contempo però non ponesse delle metaforiche sbarre tra il gruppo e il resto del mondo (una delicatezza che si può intuire dalle parole «non si mischiassero troppo») ${ }^{38}$.

D'altra parte, il contatto con il resto del mondo era proprio l'esperienza che si voleva far vivere al gruppo, con le attività in spiaggia già descritte per il 1969 in «Alba Serena», ma anche con delle passeggiate in paese e nella campagna circostante, acquisti di accessori per il nuoto e tappe per mangiare gelato e altri dolci. Durante quell'estate a Gatteo a Mare, il personale era molto più soddisfatto della «vita fuori della colonia», con gli stessi bambini attenti a conservare l'ordine per garantire la sicurezza. Ideali per un'esperienza sensoriale variegata e costruttiva erano proprio le gite in campagna, durante le quali al gruppo impossibilitato a vedere erano descritte le varietà di frutta e di verdura, oltre che le installazioni per la pesca lungo il Rubicone, organizzando giochi di ricerca nell'ambiente circostante. Anche se la pecca di fondo restava: nonostante la simpatia provata nei loro confronti dagli altri gruppi presenti nella struttura e le attività artistiche comuni nell'ultima settimana di soggiorno, «noi assistenti avremmo voluto che i nostri bambini facessero amicizia e giocassero con quelli vedenti, per sentirsi più uniti, ma non è stato possibile per non creare disordine; inoltre notavamo negli altri soltanto un interesse destato solo dalla compassione» 39 .

Tornando a considerare uno spettro cronologico più ampio per quanto riguarda l'alimentazione garantita, in genere si seguiva una dieta semplice ma nutriente. Per dare un'idea di questa offerta, a metà anni Cinquanta si proponeva: al mattino, latte con caffè o con cacao,

\footnotetext{
38 ASICMi, Assistenza e Istruzione (ex Beneficenza), Colonie estive, b. 2, fasc. 15, report per la colonia marina 1969, cit., foglio 1 recto e verso.

39 ASICMi, Assistenza e Istruzione (ex Beneficenza), Colonie estive, b. 2, fasc. 15, report per la colonia marina 1969, cit., foglio 1 verso e foglio 2 recto.
} 
accompagnato da pane; a mezzogiorno, minestra asciutta, carne o pesce e contorno di verdura, frutta fresca; a merenda, pane e marmellata o frutta; a cena, minestra in brodo o minestrone, un secondo vario con contorno, frutta fresca e pane ${ }^{40}$. Non si lesinava sulle eccezioni nell'intento di variegare e personalizzare quanto veniva offerto al palato: oltre alle lasagne e agli arrosti di cui già si è scritto41, erano previste anche spese aggiuntive per gelati, pizze e altre evasioni culinarie ${ }^{42}$. L'attenzione del personale d'accompagnamento non era allentata nemmeno in questa direzione e si interveniva subito laddove fosse necessario. Infatti, nel fatidico report del 1969 relativo alla colonia marina è possibile leggere che «il vitto era scarso e pure scadente; si lamentavano di questo soprattutto i più grandi»: soltanto l'intervento della persona scrivente riuscì a procurare una maggiore abbondanza nelle porzioni 43 .

Infine, per quanto riguarda il corredo dei ragazzini «da portare raccolto in una valigia», alla famiglia erano date delle liste dettagliate e molto precise al riguardo, così da evitare qualsiasi difficoltà su questo versante durante il periodo di vacanza. Prendendo come esempio il corredo per San Mauro a Mare nel 1955, per quasi un mese di colonia (dal 13 luglio all'11 agosto) si chiedeva di preparare: due paia di mutande, due pigiami, due magliette leggere, un cappello bianco, un golf, due mutandine da bagno, un paio di zoccoli o sandali, un paio di scarpe, tre paia di calze, quattro fazzoletti, due asciugamani, due paia di calzoncini e per le femmine due grembiulini con le maniche, di cui uno bianco. Completava la lista il necessario per la pulizia personale e una tenuta con cui presentarsi il giorno della partenza che sostituisse la divisa, quell'anno non prevista; «compatibilmente con le proprie possibilità», quindi, al ritrovo presso le scuole comunali di via Galvani - vicino alla stazione centrale di Milano - le femmine dovevano avere grembiule e cappellino bianchi, i maschi calzoncini scuri, maglietta chiara e anche loro cappellino bianco44.

Per chi fosse diretto ad Asiago, invece, erano previsti per il periodo dall'11 agosto al 6 settembre: un abito da passeggio, due paia di calzoni o sottane, due camicie o sottovesti, tre paia di mutande, un pullover o golfino, due canottiere, sei fazzoletti, due paia di scarpe e, ça va sans dire, il necessario per la toletta 45 .

\footnotetext{
40 ASICMi, Assistenza e Istruzione (ex Beneficenza), Colonie estive, b. 1, fasc. 1, brochure dell'Associazione Nazionale Combattenti e Reduci per le colonie estive 1954, cit.

${ }^{41}$ Alba Serena, dicembre 1962, cit., p. 18.

$4^{2} \mathrm{~A}$ titolo di esempio, si propone ASICMi, Assistenza e Istruzione (ex Beneficenza), Colonie estive, b. 2, fasc. 14, distinta delle spese sostenute a Gatteo a Mare nel luglio 1967, 14 settembre 1968. Nello stesso elenco, si notano anche spese per crema solare e zampironi, oltre che prestiti ai bambini per comprare francobolli e giocattoli.

43 ASICMi, Assistenza e Istruzione (ex Beneficenza), Colonie estive, b. 2, fasc. 15, report per la colonia marina 1969, cit.

44 ASICMi, Assistenza e Istruzione (ex Beneficenza), Colonie estive, b. 1, fasc. 1, lettera dell'Istituto ai genitori, 5 luglio 1955.

45 ASICMi, Assistenza e Istruzione (ex Beneficenza), Colonie estive, b. 1, fasc. 1, lettera dell'Istituto ai
} 
Per concludere l'analisi che ha tenuto conto delle attività ricreative e dell'attenzione dedicata all'alimentazione e all'igiene dei ragazzi, i dati studiati consentono di evidenziare l'ampia e variegata offerta dell'Istituto e degli enti con cui collaborava con l'intento di soddisfare tutti i requisiti per vacanze sì formative ma anche rilassanti e divertenti. Emerge un particolare riguardo nei confronti della formazione esperienziale dei giovani ciechi, da sempre una delle caratteristiche principali dell'Istituto di Milano, attento sin dai suoi albori ai metodi pedagogici e di crescita personale più innovativi e inclusivi. Nelle circostanze in cui succedeva invece che, per un eccessivo numero di giovani ospiti o per inadeguatezze architettoniche dell'ambiente, la collaborazione tra il personale di accompagnamento e di assistenza dell'Istituto e la direzione della struttura ospitante non riusciva a essere ottimale, il primo cercava di porre rimedio in maniera creativa, competente e diplomatica, così da garantire il meglio alla gioventù che gli era stata affidata cercando di coniugare sicurezza ed esperienza educativa e contribuendo a un clima di inclusione.

\section{Gestione e finanziamento delle colonie estive}

All'inizio del giugno 1973, il comitato dei genitori scriveva al consiglio d'amministrazione dell'Istituto per sollecitare risposte in merito all'organizzazione delle colonie; inoltre, domandava senza giri di parole con quanto avrebbero contribuito l'Istituto, le Province, le Regioni, le Dame Benefattrici, e quanto sarebbe stato chiesto alle famiglie dei ragazzi beneficiari del soggiorno estivo46.

Con altrettanta schiettezza, il consiglio d'amministrazione avrebbe risposto che nelle finalità fissate nello statuto di via Vivaio non rientrava «l'obbligo dell'organizzazione di colonie e soggiorni estivi [...] di competenza degli Enti Locali Assistenziali» e che l'Istituto stesso era «scolastico con compiti di assistenza limitati al periodo della Scuola». Aggiungeva però di essere sempre andato incontro alle famiglie più in difficoltà trovando il modo, «in collaborazione con gli Enti Locali e nei limiti delle proprie possibilità», di organizzare soggiorni estivi che potessero giovare al benessere degli ospiti più bisognosi dal punto di vista medico. Precisava inoltre che, per gli alunni fermi in convitto già durante i mesi scolastici, trascorrere almeno l'estate con la famiglia - laddove possibile - era molto importante «ai fini di un corretto sviluppo sul piano psicologico, affettivo, sociale» 47 .

genitori con allegato il dettaglio del corredo, 2 luglio 1955.

46 ASICMi, Assistenza e Istruzione (ex Beneficenza), Rettori, b. 4, fasc. 25, il comitato dei genitori alla direzione dell'Istituto, 6 giugno 1973.

47 ASICMi, Assistenza e Istruzione (ex Beneficenza), Rettori, b. 4, fasc. 25, comunicazione del consiglio d'amministrazione dell'Istituto relativa ai soggiorni estivi 1973, senza data. 
Seguiva comunque l'offerta di quell'anno (tabb. 1A e 1B), come sempre disponibile sia al mare che in montagna, corredata di date e ulteriori dettagli. Attenzione, però: era fatto un discrimine a seconda che i giovani ospiti fossero minori o maggiori di dodici anni. Nel primo caso, qualora la destinazione prescritta fosse balneare, era disponibile la colonia dell'ODA a Cesenatico; se invece la famiglia non intendeva usufruirne e organizzarsi diversamente, l'Istituto concedeva un rimborso del $60 \%$ sulle spese per il solo bambino - debitamente documentate - e comunque per un soggiorno non superiore ai venti giorni. Per la montagna, invece, era consigliato alle famiglie di organizzarsi per conto proprio sapendo che l'Istituto avrebbe potuto provvedere a un rimborso dell' $80 \%$ delle spese, sempre a condizione che venissero documentate e che il soggiorno non eccedesse i venti giorni; era menzionata un'anonima eccezione, in possesso di prescrizione medica del responsabile sanitario di via Vivaio, situazione della quale si sarebbe occupato finanziariamente l'Istituto stesso.

Qualora invece si trattasse di ragazzi superiori ai dodici anni, la situazione era ribaltata. Restava disponibile la pensione Stella Montis - registrata erroneamente come «Regina Montis» - a Campitello di Fassa per un soggiorno organizzato dal MAC (Movimento Apostolico Ciechi); l'Istituto dei Ciechi di Milano si assumeva «l'onere e la spesa, salvo diritti di rivalsa presso le Amministrazioni Provinciali di competenza». Ancora una volta, qualora la famiglia non intendesse servirsi dell'offerta programmata, era comunque predisposto un rimborso del $60 \%$ se rispettate le condizioni suddette. A scanso di equivoci in chiusura si precisava che, sia per il mare che per la montagna, i contributi messi a disposizione dall'Istituto non avrebbero potuto essere presi dalla retta annuale «notoriamente insufficiente» fornita dalle Province; ci si sarebbe quindi serviti di «integrazioni speciali» richieste a enti locali e al comitato delle Dame Patronesse - le stesse che il comitato dei genitori aveva chiamato «Dame Benefattrici» - dell'Istituto di via Vivaio 48 .

Questo botta e risposta, in verità, non era rappresentativo di un nuovo modo di organizzare e finanziare le colonie estive. Infatti i finanziamenti, totali o parziali che fossero, erano sempre arrivati da svariati enti: molto attive in questo senso erano state negli anni la UIC - soprattutto la sezione di Brescia -, la Provincia di Milano, quella di Mantova, talvolta i Comuni di provenienza, soprattutto dalla seconda metà degli anni Sessanta anche la Prefettura di Milano. Inoltre, in alcuni rari casi era stata l'industria in cui era impiegato un genitore del fanciullo cieco ad aver contribuito al soggiorno estivo, probabilmente in una politica di supporto benefico che interessava i figli di tutti gli operai, disabili e non. Non era nemmeno raro che l'Istituto

\footnotetext{
48 Ibidem.
} 
provvedesse alle spese di colonia estiva dei ragazzi provenienti dalle famiglie più economicamente disagiate, ricorrendo alle Dame o a fondi raccolti con altre iniziative benefiche 49 .

Anche per quest'ultimo caso «Alba Serena» si è rivelata una fonte molto utile. Infatti, nei numeri 2 e 3 del 1960 è possibile leggere rispettivamente l'annuncio e il resoconto dell'annuale fiera benefica organizzata dalle Dame dell'Istituto per contribuire al finanziamento delle colonie estive. Per quell'anno, la fiera si tenne dal 31 marzo al 3 aprile ${ }^{50}$. Nell'ampia sala Barozzi furono esposti oggetti artigianali provenienti dai vari laboratori dell'Istituto, come pizzi, tovagliati, porcellane e articoli per la casa, venduti dalle signore stesse; fiore all'occhiello fu la sezione di antiquariato, con pregevoli mobili ottocenteschi. Il bilancio venne giudicato positivo ${ }^{51}$.

Per gli ultimi anni studiati in questo articolo, si registra anche un cambiamento di fondamentale importanza: il subentrare, a partire dal 1972, della neonata Regione Lombardia e nello specifico dell'assessorato all'assistenza tra i finanziatori delle colonie estive dell'Istituto52. La presenza della Regione in tale ambito assunse rapidamente un'importanza tale che per esempio nel 1975, in virtù della legge regionale 3 settembre 1974, n. 56 (Norme relative al servizio sociale per $i$ soggiorni di vacanza dei minori) il cui art. 56 prevedeva un fondo «a favore di iniziative per le vacanze di minori affetti da carenze psico-fisiche nonché per iniziative particolari», stanziò un'ingente somma per il soggiorno di vacanza di quell'estate53.

Per avere un quadro più approfondito in merito ai finanziatori coinvolti, si veda la tab. 2 .

\footnotetext{
49 Come per le mete dei soggiorni estivi, anche la suddivisione dei finanziatori è ricavabile dallo spoglio dei documenti in bb. 1-2, fascc. 1-21.

5o Alba Serena, XXXVIII, 2, marzo - aprile 1960, p. 11.

${ }^{1}$ Alba Serena, XXXVIII, 3, maggio 1960, p. 11.

${ }^{2}$ ASICMi, Assistenza e Istruzione (ex Beneficenza), Colonie estive, b. 2, fascc. 17-21.

53 Si vedano: Bollettino Ufficiale Regione Lombardia, n. 36, $2^{\circ}$ suppl. del 06 settembre 1974; ASICMi, Assistenza e Istruzione (ex Beneficenza), Colonie estive, b. 2, fasc. 19, l'assessore ai servizi sociali della Regione al presidente dell'Istituto dei Ciechi, 8 luglio 1975.
} 
Tab. 2

\begin{tabular}{|c|c|c|}
\hline \multicolumn{3}{|c|}{ Colonie al mare } \\
\hline Finanziatore & Anno & Soggiorni pagati \\
\hline \multirow{6}{*}{ Famiglie } & 1955 & 7 \\
\hline & 1956 & 8 \\
\hline & 1957 & 5 \\
\hline & 1958 & 4 \\
\hline & 1959 & $1 \mathrm{e}$ un parziale \\
\hline & 1960 & 4 \\
\hline \multirow{3}{*}{ Istituto dei Ciechi } & 1955 & 1 \\
\hline & 1957 & 2 \\
\hline & 1960 & 7 \\
\hline \multirow{6}{*}{ Sedi UIC competenti } & 1955 & 1 \\
\hline & 1956 & 2 \\
\hline & 1957 & 2 \\
\hline & 1958 & 1 \\
\hline & 1959 & 4 \\
\hline & 1960 & 1 \\
\hline \multirow{6}{*}{$\begin{array}{l}\text { Comuni di } \\
\text { provenienza }\end{array}$} & 1955 & 6 \\
\hline & 1956 & 7 \\
\hline & 1957 & 3 e un parziale \\
\hline & 1958 & 4 \\
\hline & 1959 & 2 \\
\hline & 1960 & 3 \\
\hline \multirow{4}{*}{$\begin{array}{l}\text { Province di } \\
\text { provenienza }\end{array}$} & 1957 & $1 \mathrm{e}$ un parziale \\
\hline & 1960 & 1 \\
\hline & 1961 & 14 \\
\hline & 1962 & 10 \\
\hline $\begin{array}{l}\text { Prefetture } \\
\text { competenti }\end{array}$ & 1957 & un parziale \\
\hline \multirow{3}{*}{$\begin{array}{l}\text { Datori di lavoro dei } \\
\text { genitori }\end{array}$} & 1956 & 1 \\
\hline & 1957 & 1 \\
\hline & 1959 & un parziale \\
\hline \multirow{6}{*}{ Non precisato } & 1955 & 2 \\
\hline & 1956 & 2 \\
\hline & 1957 & 3 \\
\hline & 1958 & 2 \\
\hline & 1959 & 4 \\
\hline & 1960 & 9 \\
\hline
\end{tabular}

\begin{tabular}{|c|c|c|}
\hline \multicolumn{3}{|c|}{ Colonie in montagna } \\
\hline Finanziatore & Anno & $\begin{array}{c}\text { Soggiorni } \\
\text { pagati }\end{array}$ \\
\hline \multirow{4}{*}{ Famiglie } & 1956 & 2 \\
\hline & 1957 & 2 \\
\hline & 1958 & 2 \\
\hline & 1959 & 1 \\
\hline \multirow{2}{*}{ Istituto dei Ciechi } & 1957 & 1 \\
\hline & 1958 & 1 \\
\hline \multirow{5}{*}{ Sedi UIC competenti } & 1955 & 5 \\
\hline & 1956 & 1 \\
\hline & 1957 & 3 e un parziale \\
\hline & 1958 & 1 \\
\hline & 1959 & 1 \\
\hline \multirow{5}{*}{ Comuni di provenienza } & 1955 & 3 \\
\hline & 1956 & 3 \\
\hline & 1957 & 2 e un parziale \\
\hline & 1958 & 4 \\
\hline & 1959 & 3 \\
\hline \multirow{3}{*}{ Province di provenienza } & 1959 & 1 \\
\hline & 1961 & 5 \\
\hline & 1962 & 7 \\
\hline \multirow{2}{*}{ Non precisato } & 1958 & 3 \\
\hline & 1959 & 5 \\
\hline
\end{tabular}

Fonte: ASICMi, Assistenza e Istruzione (ex Beneficenza), Colonie estive, b. 1-2, fascc. 1-21; Rettori, b. 4, fasc. 25. 


\section{Suggestioni per studi futuri}

Lo studio delle colonie estive organizzate dall'Istituto dei Ciechi di Milano e dagli enti che con esso collaboravano è stato qui inserito in un quadro che ha cercato di tenere conto sia della percezione della persona con disabilità visiva nell'Italia dei decenni Cinquanta-Settanta del Novecento, sia del background culturale e pedagogico dell'istituzione milanese considerata, sia della rete assistenziale a cui quest'ultima faceva riferimento con i dovuti cambiamenti in base all'evolversi della società e, soprattutto negli Settanta, della normativa italiana.

Una caratteristica che è emersa dalla gestione delle colonie estive - una prima forma di turismo inclusivo - da parte dell'Istituto è l'iniziativa di proporre agli assistiti e alle assistite lo spettro più ampio possibile di interazione con quello che si potrebbe considerare il «mondo esterno", cercando in tutti i modi di gestire in maniera soddisfacente per tutti le inevitabili difficoltà dovute alla scarsa conoscenza delle disabilità da parte della società italiana in quei decenni e alle barriere architettoniche ancora lungi dall'essere affrontate in maniera più diretta dalla normativa successiva. Dallo studio della documentazione d'archivio, coronata dal report del 1969, e dall'analisi critica delle fonti a stampa di «Alba Serena», si è giunti alla conclusione che l'Istituto e alcuni degli enti con i quali collaborava in occasione dell'estate proponevano alla gioventù di cui erano responsabili un turismo postmoderno ante litteram. Si trattava, infatti, di una vera e propria forma di turismo «esperienziale» che, se da una parte superava letteralmente i vincoli imposti dall'ambiente antropico, dall'altra parte per contro faceva proprie le caratteristiche del territorio - in questo caso sia naturali che antropiche - per creare un vero e proprio laboratorio a cielo aperto, in cui i giovani e le giovani potevano fondersi con lo scenario circostante pur essendo impossibilitati a fruirlo con il senso della vista: dalle campagne del Rubicone al litorale tirrenico, dall'altopiano di Asiago alle strade di Pisa.

Pertanto, in un periodo in cui secondo i paradigmi storiografici accettati si passava dal turismo moderno al turismo di massa, l'Istituto dei Ciechi proseguiva l'esperienza montessoriana e la riproponeva in chiave turistico-esperienziale, offrendo occasioni di turismo postmoderno ai propri ospiti con un discreto anticipo rispetto, per esempio, alla riscoperta del valore culturale e artistico dei centri storici a partire dagli anni Settanta o ai percorsi letterari, ai pacchetti benessere e ai sentieri della natura dagli anni Ottanta 54 .

Certo, è necessario tenere conto che si trattava di una proposta ad hoc, per un'utenza ristretta e che altrimenti avrebbe avuto serie difficoltà a trascorrere in maniera rilassante e ricreativa il monotono periodo dell'estate: un'utenza con uno specifico tipo di disabilità, che le normali

54 Per questi filoni del turismo postmoderno allora in costruzione, si rimanda soprattutto a BATTILANI, Patrizia, op. cit., pp. 160-162. Utile anche un confronto con ROCCA, Giuseppe, Dal prototurismo al turismo globale, cit., pp. 104-115. 
infrastrutture turistiche non erano pronte ad accogliere. Tuttavia, se alcune delle caratteristiche individuate dagli studiosi per il turismo postmoderno sono proprio la soggettività e il desiderio di un'esperienza diversa da quella proposta alla massa, ecco che in maniera armonica a fianco dei tentativi di interazione e inclusione queste caratteristiche di unicità e rottura emergono qua e là nell'organizzazione estiva delle colonie studiate.

Già nel 1979 Erik Cohen55, come ha evidenziato Patrizia Battilani56, ragionava sulle nuove esigenze della comunità di viaggiatori e individuava differenti modalità di vivere le esperienze turistiche, notando una crescente rottura con la tradizione di viaggio ormai consolidata e un interesse maggiore nei confronti della sperimentazione. Negli anni successivi il dibattito sarebbe continuato sia dal punto di vista storiografico sia - e soprattutto - dal punto di vista sociologico e antropologico, approfondendo la caratteristiche del nuovo tipo di turismo imperante ${ }^{57}$. Nel turismo italiano, però, l'esempio delle colonie estive dell'Istituto dei Ciechi si presenta con la caratteristica aggiunta di affiancare a una forma antesignana di turismo «esperienziale» lo sforzo teso verso l'inclusione e l'interazione delle persone cieche con il resto della società, nonostante i numerosi ostacoli.

L'articolo ha quindi voluto presentare questo aspetto inedito dell'Istituto di via Vivaio, cercando di porre le basi per la discussione e il confronto con futuri studi in questo ambito di ricerca, in modo che si possa apportare un contributo alla storia del turismo e alla storia dell'inclusione delle persone disabili.

\footnotetext{
55 COHEN, Erik, «A phenomenology of tourist experiences», in Sociology, 13, 2/1979, pp. 179-201.

${ }_{56}^{6}$ BATTILANI, Patrizia, op. cit., pp. 161-162, in particolare la nota 16.

57 Si segnalano alcuni dei contributi più significativi: FEIFER, Maxine, Going places. The ways of the tourist from Imperial Rome to the present day, London, MacMillan, 1985; URRY, John, The tourist gaze: leisure and travel in contemporary societies, London, Sage, 1990; MUNT, Ian, «The "other" postmodern tourism: culture, travel and the new middle classes», in Theory, Culture and Society, 11, 3/1994, pp. 101-123; MINCA, Claudio, Spazi effimeri. Geografia e turismo tra moderno e postmoderno, Padova, Cedam, 1996; ID., «(De)costruire lo spazio turistico», in Bollettino della Società Geografica Italiana, 4, 2/1997, pp. 511-522; ECHTNER, Charlotte M., JAMAL, Tazim B., op. cit.; URIELY, Natan, "Theories of modern and postmodern tourism», in Annals of Tourism Research, 24, 4/1997, pp. 892-895; ID., «The tourist experience: conceptual developments», in Annals of Tourism Research, 32, 1/2005, pp. 199-216; WILLIAMS, Stephen, LEW, Alan A., Tourism geography. Critical understandings of place, space and experience, London, Routledge, 2014.
} 


\section{GLI AUTORI}

Luciano MAFFI è dottore di ricerca in storia moderna e contemporanea. Attualmente è assegnista di ricerca di storia economica presso l'Università degli studi di Genova e insegna storia del turismo presso l'Università Cattolica del Sacro Cuore. I suoi studi riguardano la storia del turismo, soprattutto in relazione ai cambiamenti infrastrutturali ed economici dell'Ottocento e del Novecento. Si occupa inoltre di sistemi di assistenza, di sistemi creditizi e finanziari e di produzione alimentare nella storia moderna e contemporanea.

URL: < http://www.studistorici.com/progett/autori/\#Maffi >

Martino Lorenzo FAGNANI è dottorando in storia moderna e contemporanea presso l'Università degli studi di Pavia e si è laureato in cultura e storia del sistema editoriale presso l'Università degli studi di Milano. Nell'ambito della ricerca, attualmente studia temi di storia della scienza nel Settecento e nell'Ottocento. I suoi studi abbracciano anche la storia culturale e la storia del turismo nel corso dell'Ottocento.

URL: < http://www.studistorici.com/progett/autori/\#Fagnani > 